\title{
INTRAVASCULAR ULTRASOUND ELASTOGRAPHY IN HUMAN ARTERIES: INITIAL EXPERIENCE IN VITRO
}

\author{
Chris L. de Korte, Antonius F. W. van der Steen, E. Ignacio Céspedes and \\ GeRARD PASTERKAMP \\ Experimental Echocardiography, Thoraxcenter, Erasmus University Rotterdam, Rotterdam, The Netherlands; \\ Interuniversity Cardiology Institute of the Netherlands, Utrecht, The Netherlands; EndoSonics Corporation, Rancho \\ Cordova, CA USA; and Department of Functional Anatomy, Utrecht University, Utrecht, The Netherlands
}

\author{
(Received 24 July 1997; in final form 11 November 1997)
}

\begin{abstract}
Intravascular elastography is a new technique to obtain the local mechanical properties of the vessel wall and its pathology using intravascular ultrasound (IVUS). Knowledge of these mechanical properties may be useful for guiding interventional procedures. An experimental set-up is described for assessment of the strain data of arteries. Using a 30-MHz IVUS catheter, radio frequency data are acquired with a custom-made high-performance data acquisition system. High-resolution, local tissue displacement estimation by crosscorrelation is followed by computation of local strain. An algorithm that uses a priori knowledge of the correlation coefficient function was applied to filter the obtained strain data. With this experimental set-up, intravascular elastograms containing 400 angles/revolution with a radial resolution of $200 \mu \mathrm{m}$ can be produced. The feasibility of intravascular elastography with this experimental set-up is demonstrated using two diseased human femoral arteries. Qualitative comparison of the elastograms with the echograms and the histology demonstrates the potential of intravascular elastography to obtain mechanical information from the vessel wall and from plaque. (C) 1998 World Federation for Ultrasound in Medicine \& Biology.
\end{abstract}

Key Words: Intravascular ultrasound elastography, Strain imaging, Human arteries, Plaque characterisation, Elasticity

\section{INTRODUCTION}

Despite a significant decline in the last 25 years, atherosclerotic coronary disease remains the leading cause of death in the Western world. Physicians need to select the most appropriate technique for treating patients suffering from atherosclerotic disease. As the number of available interventional techniques for treatment of atherosclerotic luminal narrowing increases, the specific diagnostic information becomes increasingly important. Advances in high-frequency intravascular ultrasound (IVUS) have made it possible to study the morphology of the vessel wall and its pathology. Currently, IVUS is the only clinically available technique capable of providing realtime cross-sectional images in vivo, delivering information that is not available from $\mathrm{x}$-ray angiography. For this reason, IVUS is being used more routinely for guiding and selecting interventional procedures (Fitzgerald and

Address correspondence to: Chris L. de Korte, Experimental Echocardiography, Ee 23.02, Thoraxcenter, Erasmus University Rotterdam, P.O. Box 1738, 3000 DR Rotterdam, The Netherlands.
Yock 1993; Isner et al. 1991, Mintz et al. 1994), for investigation of the effectiveness of the procedure (Baptista et al. 1996; Gussenhoven et al. 1995) and for studying the mechanisms for restenosis (Mintz et al. 1996; von Birgelen et al. 1995).

Since the outcome of the interventional procedure is determined not only by the morphology of the diseased vessel but also by the tissue components of the atheroma (Fitzgerald and Yock 1993; Honye et al. 1992), knowledge of these properties is useful. Characterisation of the atherosclerotic plaques using IVUS is still limited. Using IVUS, calcified deposits can be identified in most cases due to the bright echo of this material and the distal acoustic shadow (Wolverson et al. 1983); however, identification of fatty, fibro-fatty and fibrous tissue is much more complex. Using the spectral information of the radio frequency (rf) signal, it is possible to discriminate between some types of atheroma in vitro (Barzilai et al. 1987; Bridal et al. 1997a, 1997b; Landini et al. 1986; Spencer et al. 1997; Wilson et al. 1994), but the feasibility in vivo has not yet been shown. 
Knowledge of the mechanical properties of the vessel wall and the atherosclerotic lesions may be even more important than characterisation of the different plaque types. Lee and coworkers demonstrated the predictability of locations of plaque fracture as a result of balloon angioplasty (Cheng et al. 1993; Lee et al. 1993). They suggest that concentrations of stress can occur at junctions between hard material (plaque) and softer material (vessel wall). These high stress regions may predispose to plaque fracture.

IVUS can be used to study the mechanical properties of vascular tissue. Measuring the change in lumen area under application of a differential intraluminal pressure, the distensibility of the vessel can be determined from inside using IVUS (The et al. 1995). In some applications (e.g., carotid artery), the distensibility also can be determined transcutaneously using echo-tracking techniques to determine the diameter of the vessel (Hoeks et al. 1992; Mozersky et al. 1972; Pagani et al. 1979) or using echo-Doppler techniques (Reneman et al. 1986). We describe an improved technique to determine the local mechanical properties of the vessel wall with atherosclerotic disease. This technique is based on the elastography principle proposed by Ophir et al. (1991) and applied in vivo by Céspedes et al. (1993) for nonvascular applications. Using this technique, an elastogram (an image of strain or elastic modulus) is formed. Recent phantom studies revealed that this technique also may be applicable for intravascular purposes (de Korte et al. 1997; Ryan and Foster 1997a, 1997b; Shapo et al. 1996). In gelatin-based vessel-mimicking phantoms with the morphology of vessels containing hard or soft plaques, regions with different elastic properties could be identified using the elastogram, independent of the corresponding echogenicity contrast. Although we were able to demonstrate the principle of intravascular elastography on slightly upscaled phantoms, the spatial resolution of the technique was not suitable for imaging in real vessels. Using phantoms with a wall thickness of $5 \mathrm{~mm}$, strain estimates were obtained for each $400 \mu \mathrm{m}$. However, this resolution is insufficient to produce elastograms of real artery specimens with a wall thickness that is normally $1 \mathrm{~mm}$ or less. In this study, a technique with improved resolution is described. Using a new experimental set-up and a custom-made data acquisition system, the signal-to-noise ratio was increased and motion artifacts were minimised. Additionally, new signal processing procedures were implemented to improve the quality of the strain values obtained. With these modifications, the resolution of the elasticity images is improved to a suitable level for elastography in arteries as demonstrated by the first elastograms of human femoral arteries in vitro. A qualitative comparison with conventional echo images, histology and compression modulus

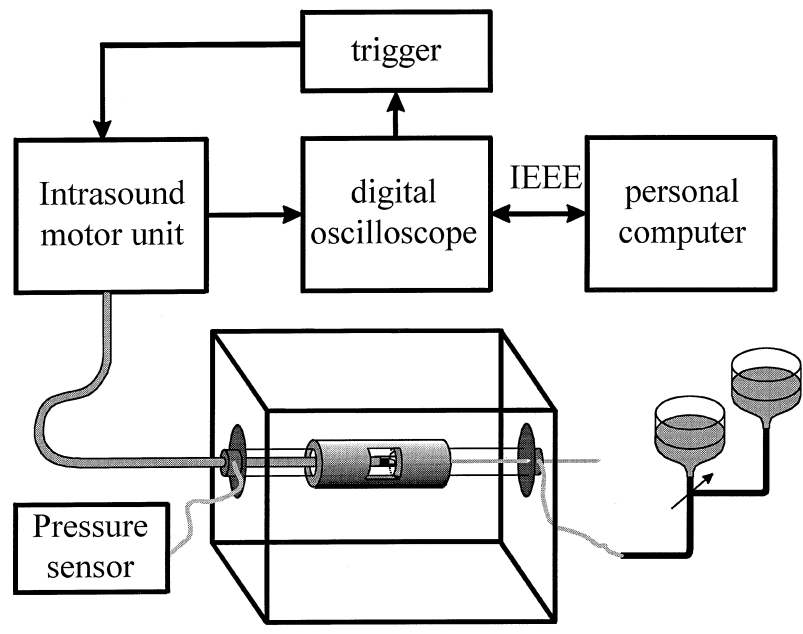

Fig. 1. Experimental set-up.

values from the literature is made to illustrate that intravascular elastography may be a useful technique to characterise mechanical properties of different plaque types.

\section{METHODS}

\section{Data acquisition}

The experiments were performed in a water tank equipped with two insertion sheaths (8 Fr) at either side (Fig. 1), using a modified $4.3 \mathrm{~F}$ Princeps ${ }^{\circledR} 30-\mathrm{MHz}$ catheter (DuMED [currently EndoSonics Europe], Rijswijk, The Netherlands). The original, flexible, drive shaft of the catheter was replaced by a rigid tube (diameter $0.85 \mathrm{~mm}$, length $150 \mathrm{~mm}$ ) to avoid motion artifacts due to nonuniform rotation of the transducer (ten Hoff et al. 1989). The IVUS catheter was inserted via the proximal sheath. Intraluminal pressure was applied by a water column system containing degassed physiological saline solution connected to a side arm of the proximal sheath. The intraluminal pressure was monitored using a pressure gauge (DTX/plus ${ }^{\circledR}$ Ohmeda, Bilthoven, The Netherlands) connected to a side arm of the distal sheath.

The catheter is connected to a modified Intrasound ${ }^{\circledR}$ motor unit (DuMED [currently EndoSonics Europe]). This motor unit contains the pulser and receiver of the ultrasonic system and rotates the catheter in 400 angles/ revolution using a stepper motor. At each angular position, 12 traces of $10.0 \mu$ s were acquired, representing an echo depth of $7.5 \mathrm{~mm}$. The 12 traces were averaged to improve the signal-to-noise ratio (SNR): we measured an average SNR of $26 \mathrm{~dB}$ for the first $500 \mu \mathrm{m}$ of tissue (calcified tissue excluded). Two scans were acquired, one at a pressure level of $80 \mathrm{mmHg}$ and one at 100 $\mathrm{mmHg}$.

The rf data were stored in a custom-made acquisi- 
tion system. This system contains an industrial grade Pentium $^{\circledR}$ 133-MHz computer, equipped with two DA500A data acquisition boards (Signatec, Corona, CA, USA). The rf signals were digitised at $200 \mathrm{MHz}$ in 8 bits and stored in a high-speed memory of $128 \mathrm{MB}$. For phase-synchronous sampling, triggering of the ultrasonic system was synchronised with the external sampling clock output of the acquisition board. Using a lowfrequency data acquisition board (ASO 1800, Keithley, USA), the electronic output of the pressure sensor was sampled at $100 \mathrm{kHz}$. The data were processed off-line.

\section{Data processing}

The portion of the signal containing data from the vessel wall was determined. The starting and ending points of the vessel wall for each trace were detected using a threshold value just above the noise level of the system. These starting and ending point values were filtered in the angular direction using a 15-point median filter.

Successive windows of 100 samples $(T=0.5 \mu \mathrm{s})$ with $50 \%$ overlap were taken to determine the local time shifts between the traces acquired at the different pressure levels. The time shifts were determined using the peak of the correlation coefficient function. The correlation coefficient function was upsampled by a factor of 50 using a low-pass interpolation algorithm (IEEE 1979) to achieve a time shift resolution of 100 ps. A value of 100 ps is on the order of the Cramér-Rao lower bound for time shift estimation (Céspedes et al. 1995a) for the set-up used ( $80 \mathrm{ps}$ with $f_{c}=30 \mathrm{MHz}, B=20 \mathrm{MHz}, T=$ $500 \mathrm{~ns}$ and $\mathrm{SNR}=400$ ).

Local radial strain $\hat{\epsilon}$ was estimated using a onedimensional finite difference algorithm:

$$
\hat{\boldsymbol{\epsilon}}=\frac{\delta t_{2}-\delta t_{1}}{\Delta T}
$$

where $\delta t_{1}$ is time the shift between the two echo signals observed through a window of duration $T$ starting at a certain time $t$, and $\delta t_{2}$ is the time shift between the two echo signals observed through a window of the same duration $T$ but starting at time $t+\Delta T$. The time between successive windows $(\Delta T)$ of 250 ps results in a strain estimate for each $200 \mu \mathrm{m}$.

The strain data were filtered using the value of the peak of the correlation coefficient function as a figure of merit, as follows. First, the locations of the peaks of both correlation coefficient functions $\left(\rho_{1}\right.$ and $\left.\rho_{2}\right)$ were determined and the strain value was calculated. Next, this strain value was used to determine the theoretical peak value $\rho_{t h}$ of the involved cross-correlation functions as described by Céspedes et al. (1997):

$$
\begin{aligned}
\rho_{t h} & =\operatorname{sinc}\left(\epsilon f_{c} T\right) \\
\text { where } \epsilon & =\text { strain, } f_{c}=\text { center frequency and } \\
T & =\text { window length. }
\end{aligned}
$$

Finally, the two measured values $\left(\rho_{1}\right.$ and $\left.\rho_{2}\right)$ were compared to the theoretical peak value $\rho_{t h}$ and the strain estimate was rejected when the difference between the theoretical and one or both measured values was larger than 0.2 . The threshold value of 0.2 was taken from the $65 \%$ confidence interval (1\% strain and a time window of $0.5 \mu \mathrm{s}$ ) in the theoretical study (Céspedes et al. 1997).

Rejected strain values were replaced by the median value of the eight surrounding neighbours (the median of the five surrounding neighbours was taken at the border of the vessel wall).

\section{Imaging}

The rf signals were demodulated using the magnitude of the analytic signal to determine the envelope of the signal. Next, the 2000 samples in the radial direction of the envelope of each trace were downsampled to 100 points. The resulting values are converted to a linear grey scale and grey scales are plotted using a piecewise bilinear interpolation.

The strain value is colour coded using a "traffic light" notation: i.e., from red for stiff material via yellow to green for compliant material. The lower bound for strain estimation error (Céspedes et al. 1995b) using this experimental set-up is $0.03 \%\left(f_{c}=30 \mathrm{MHz}, B=20\right.$ $\mathrm{MHz}, T=600 \mathrm{~ns}, \Delta T=250 \mathrm{ps}$ and SNR $=400)$. Strain estimates were clipped between $0.03 \%$ (coded in red) and $1 \%$ (coded in green), with only a few unreliable estimates above the $1 \%$ threshold.

\section{Materials}

Two atherosclerotic human femoral arteries obtained postmortem were used for illustration of the method. The femoral arteries were excised within $24 \mathrm{~h}$ postmortem and stored at $-70^{\circ} \mathrm{C}$. The arteries were thawed at $4^{\circ} \mathrm{C}$ and measured at room temperature. The water tank of the experimental set-up was filled with a degassed physiological saline solution, and the proximal and distal sides of the artery specimens were connected to the sheaths. Each specimen was scanned at three positions separated by $10 \mathrm{~mm}$.

After the intravascular experiments, the vessel specimens were fixed for $12 \mathrm{~h}$ in a buffered $(\mathrm{pH}=7)$ formaldehyde solution (3.6\%). The specimens were decalcified in a standard RDO solution (Apex Inc., Plainfield, IL, USA). For histologic comparison, the arteries were processed for routine paraffin embedding. A pair of transverse sections of $5-\mu \mathrm{m}$ thickness, perpendicular to 


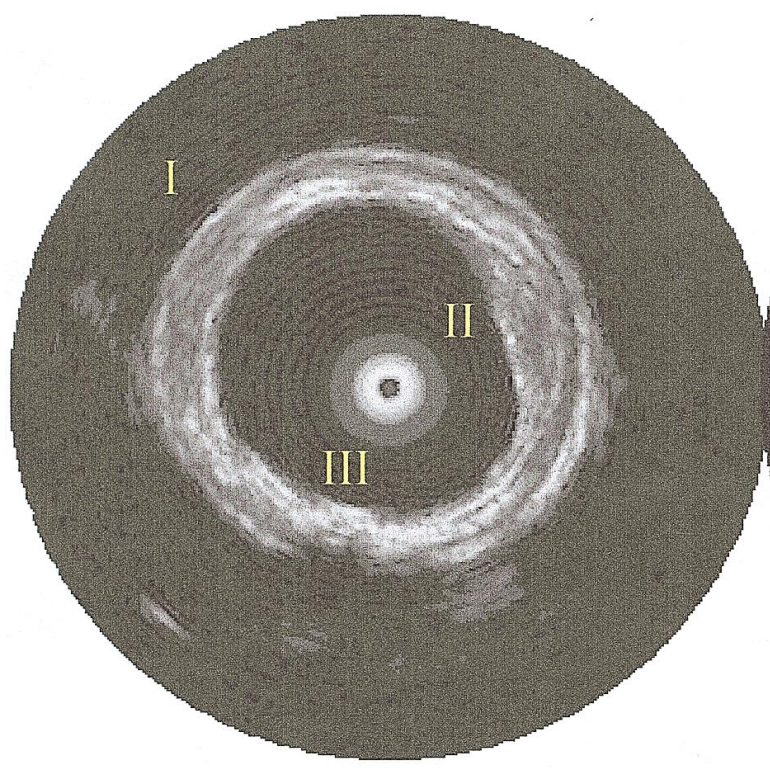

a

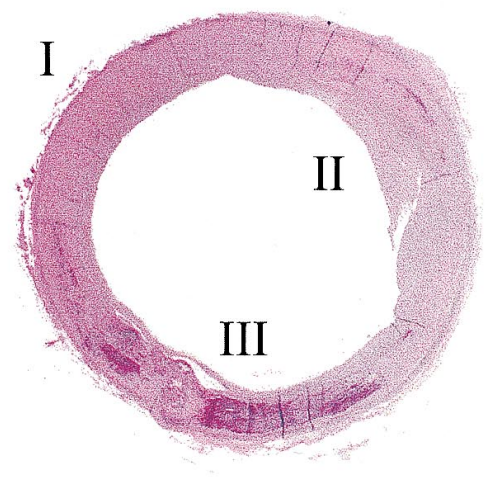

$\mathrm{c}$

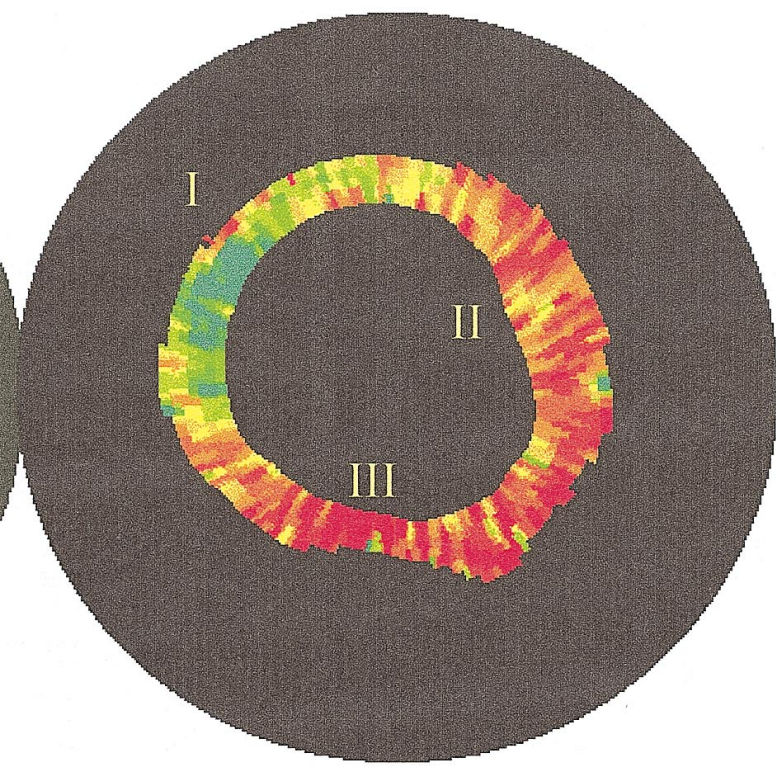

$\mathrm{b}$

$1 \mathrm{~mm}$

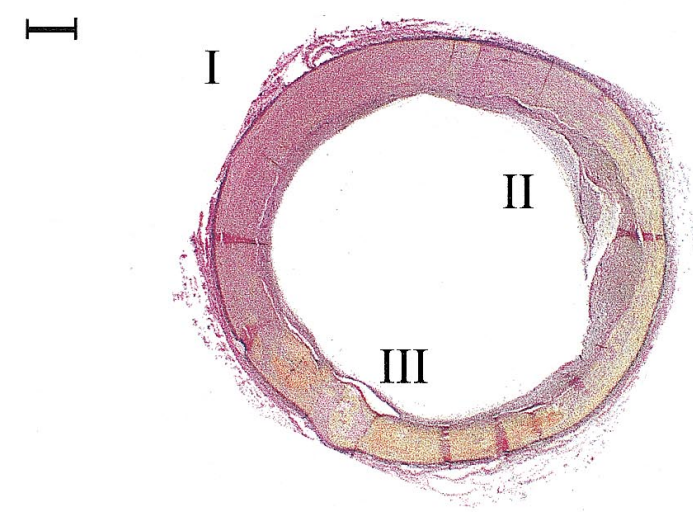

d

Fig. 2. Corresponding (a) echogram, (b) elastogram and histologic counterparts with (c) hematoxylin-eosin and (d) Verhoeff's elastic van Gieson stain of a human femoral artery. In the four images, a normal artery wall (I), a fibrous plaque deposition (II) and a calcified region (III) are apparent.

the long axis of the vessel, were cut at positions separated by $1 \mathrm{~mm}$. One section of each pair was stained with hematoxylin-eosin to assess general structural features. The remaining section was stained with the Verhoeff's elastic van Gieson, which gives selective black staining of elastin fibres. The van Gieson technique was used to counterstain muscle and connective tissue.

\section{RESULTS}

With the improved experimental set-up described here, we were able to obtain elastograms of diseased human arteries. The radial resolution of $200 \mu \mathrm{m}$ is sufficient to obtain several strain values within a normal arterial wall. In the thickened wall, up to 10 strain values are obtained.

The echogram and elastogram of a human femoral artery is shown in Fig. 2. The echogram (Fig. 2a) shows the three-layered structure of the femoral artery. A plaque deposition positioned toward the vessel wall is visible at region II. The composition of this plaque cannot be obtained from the echogram. At region III, a bright echo with distal shadowing, indicating calcified material, is apparent. The elastogram of this section is shown in Fig. 2b. Regions with very small compression are coded in red, moderately compressed material is coded yellow and tissue compressed up to $1 \%$ is coded in 


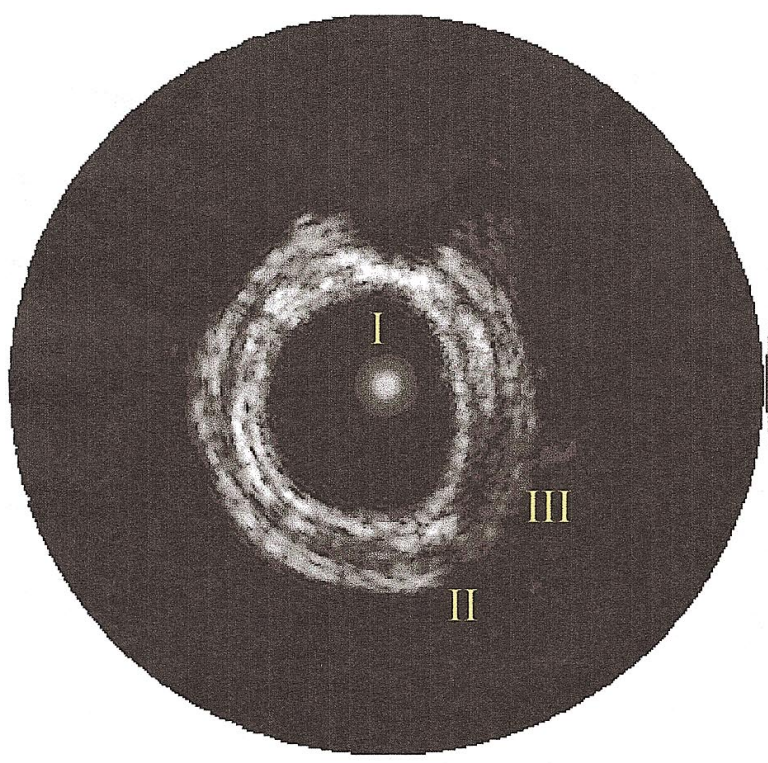

a

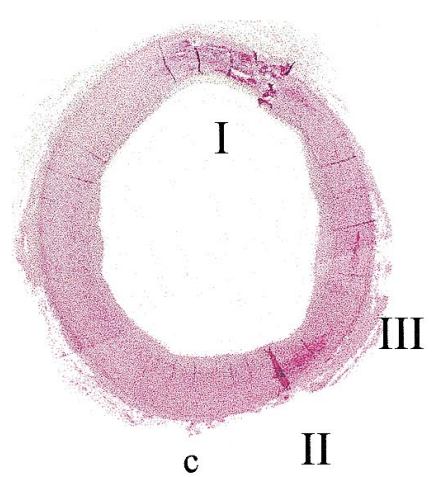

$1 \mathrm{~mm}$

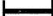

$\mapsto$

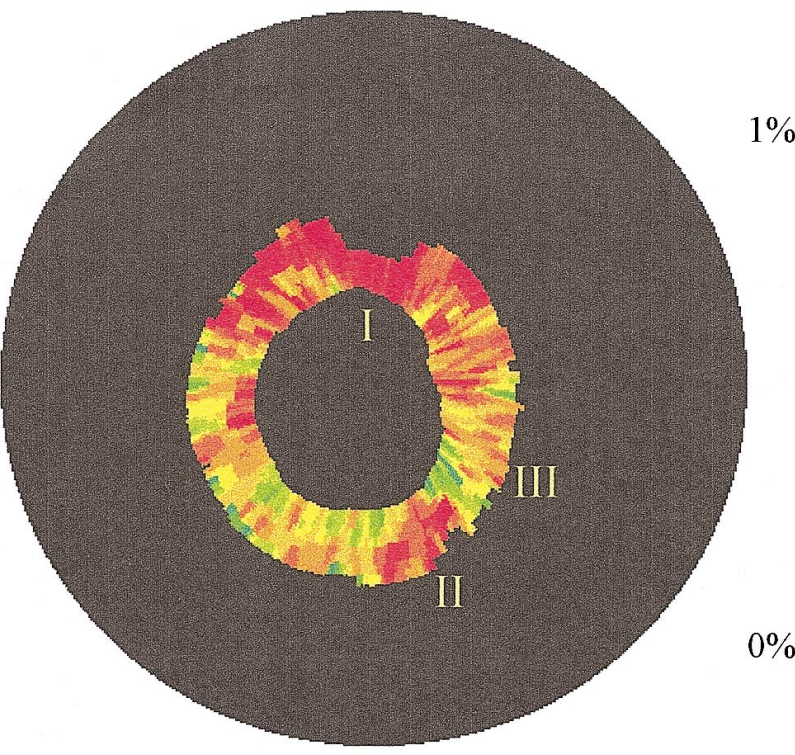

b

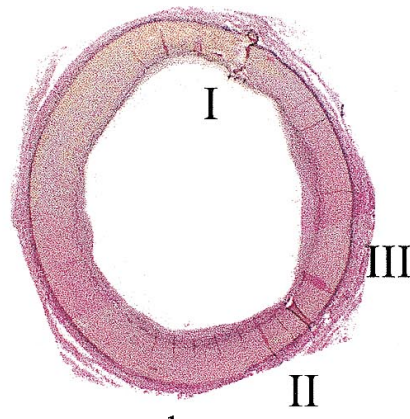

d

Fig. 3. Corresponding (a) echogram, (b) elastogram and histologic counterparts with (c) hematoxylin-eosin and (d) Verhoeff's elastic van Gieson stain of a human femoral artery. In the four images, a severe calcified region (I) and a small calcified deposit (II) are apparent. An echolucent region in the echogram is visible at region III.

green. The elastogram shows small compression in region III, indicating stiff material. The strain in region I is around $1 \%$, indicating compliant material. A moderate strain on the order of $0.25 \%$ is observed in region II.

The histologic section stained with hematoxylineosin (Fig. 2c) shows a plaque at region II. At region III, a calcified deposit can be found in the media. The rest of the vessel wall shows no plaque depositions, with only a minor formation of intimal hyperplasia. The Verhoeff's elastic van Gieson stained section (Fig. 2d) shows that the main component of the plaque at region II is fibrous material.

The result of the other femoral artery is shown in Fig. 3. The IVUS image shows calcified deposits (a bright echo with distal shadowing) at regions I and II. An echolucent media is apparent at region III. The elastogram clearly indicates the calcifications in regions I and
II, with moderate compression in other parts of the vessel wall. The strain in region III is similar to the strain in the other noncalcified parts of the vessel wall. The calcified deposits also are seen in the hematoxylin-eosin stained section where a calcified area is visible, located at the intima-media boundary in region I, and the smaller calcified spot in region II is located more distally in the media. The elastogram adequately depicts the depth of the calcified deposits within the diseased wall. The histologic sections show no difference in tissue composition at region III as compared to the other normal vessel wall tissue.

\section{DISCUSSION}

In this study, we present an improved elastographic data acquisition and signal processing method capable of 
obtaining elastograms of arteries in vitro. With respect to an earlier phantom study (de Korte et al. 1997), the flexible shaft of the Princeps ${ }^{\circledR}$ catheter was replaced by a rigid shaft to reduce motion artifacts. These motion artifacts introduce large noise components and preclude strain imaging in vessels. Rotational artifacts may be minimised by using array catheters such as the synthetic phased array catheter described by O'Donnell et al. (1997), and an interface to acquire rf signals from such devices currently is being developed.

Although the use of array catheters minimises rotational artefacts, two-dimensional search algorithms will be necessary for in vivo experiments. The pulsation of the arterial system introduces a noncontrolled movement of the catheter in the lumen, resulting in a misalignment of successive frames. Two-dimensional search algorithms currently are being implemented to advance to in vivo intravascular elastography. An integrated catheter consisting of an imaging device and a compliant balloon (Shapo et al. 1996) is another possibility to advance to in vivo elastography. Motion artifacts are minimised with this combined catheter, but blood flow is interrupted; thus, a custom device is required.

Using the new data acquisition system and averaging of the signals, the SNR is increased by $10 \mathrm{~dB}$. A better SNR allows the use of smaller time windows for the time shift estimation, resulting in an increased radial resolution of $200 \mu \mathrm{m}$. Also, a filtering technique was applied that uses a priori knowledge of the expected correlation coefficient value (Céspedes et al. 1997). Since the time shift is determined using cross-correlation, the correlation coefficient can be compared to the theoretical correlation coefficient corresponding to that strain value and selectively discarded. When the theoretical peak value differs from the determined peak values, the strain value is rejected. In this way, $20 \%$ of the strain estimates were detected to be incorrect and consequently rejected.

It was shown that elastograms of human arteries can be obtained in vitro. Using the elastogram, regions with various pathological properties can be discriminated. In both arteries, the strain in the regions containing calcified material (Fig. 2, region III and Fig. 3, regions I and II) is small, since these regions are very stiff. The strain in the normal vessel wall is up to $1 \%$, indicating softer material. The region with the fibrous plaque deposition (Fig. 2 , region II) has a moderate strain level. A radial resolution of $200 \mu \mathrm{m}$ is sufficient for adequate depiction of depth information. The elastogram (Fig. 3b) clearly shows a difference between a calcified deposit located at the intima-media interface (region I) and a deposit located in the media (region II).

In general, strain elastograms give an artifactual representation of the Young's modulus distribution.
When soft material is covered by a cap of hard material, the strain in the soft material may be decreased (Céspedes et al. 1996). This mechanical shadowing is related not only to the hardness of the materials but is dominated mainly by the geometry. In this study, the strain in the arterial wall distal to the fibrous plaque (Fig. 2, region II) is lower than the strain in the wall without plaque (Fig. 2, region I). This effect can be caused both by the radial decay of the strain (de Korte et al. 1997) and the mechanical shadowing by the fibrous plaque.

The Young's modulus is a function of the stress and the strain. Since the local strain is determined in this study, the local stress is needed for calculation of the local Young's modulus. Using this method implies that only the applied stress at the lumen-vessel wall boundary is known. For a homogeneous material, the stress and strain in the vessel wall can be calculated (de Korte et al. 1997), but this will, in general, not be the case for real artery specimens. In these cases, the use of finite element analysis can be helpful for better understanding of the strain images (de Korte et al. 1996; Soualmi et al. 1997). Nevertheless, the values for the strain of the innermost layer of the vessel wall can be converted to approximate compression modulus values. The applied stress in this layer is the intraluminal pressure differential. An intraluminal pressure of $20 \mathrm{mmHg}$ corresponds to an applied stress of $2.7 \mathrm{kPa}$. Using the relation $\sigma=\mathrm{E} \epsilon$, where $\sigma$ is applied stress, $E$ is the compression modulus and $\epsilon$ is the strain, the compression modulus for different tissue types can be calculated. This results in values of $300-600 \mathrm{kPa}$ for normal vessel wall and approximately $1 \mathrm{GPa}$ for fibrous plaque. Calcified material will have a compression modulus of several GPa. Quantitative comparison of compression moduli found in different studies is difficult, since the compression modulus is influenced by various measurement conditions (time after excision, temperature, amount of stretching). Nevertheless, the compression modulus of normal vessel wall found in this study is in the same range as values found in the literature for normal vascular tissue. Reported values range from 300-700 kPa (Bergel 1961; Gow et al. 1968; Patel et al. 1970a, 1970b). Values found for the compression modulus of atherosclerotic material span a wide range, but studies of Lee et al. $(1991,1992)$ indicate that the stiffness of calcified material is about three times higher than that of fibrous material. This finding is similar to the results of this study. The echogram reveals the geometry of the plaque (Fig. 2, region II), but the composition of the plaque remains unknown. However, based on the mechanical properties, the elastogram reveals that this plaque may be composed of fibrous tissue. This finding is corroborated by the histology.

Although the elastographic results indicate good agreement with literature values for the compression 
modulus for the various pathologies, an in vitro study with a larger number of specimens needs to be conducted to evaluate the capabilities of intravascular elastography to quantify the mechanical properties of vessel wall and plaque. For this study, staining techniques specifically suited to identify fibrous, calcified and fatty tissue are required. Due to the preliminary nature of this work, we only used staining techniques for overall morphological analysis.

\section{CONCLUSION}

Based on improved experimental and processing conditions, we have obtained the first strain elastograms of diseased arteries in vitro. Preliminary elastograms demonstrate that quantification of mechanical properties and characterisation of regions with various pathologies may be feasible. Although additional information is required to determine the exact Young's moduli, the strain elastogram reveals useful information that is inconclusive from IVUS alone. The success of this study provides motivation to initiate a larger scale in vitro study to evaluate the capabilities of IVUS elastography to quantify the mechanical properties and characterise plaque constituents. Much additional work is required to advance this technique to in vivo imaging.

Acknowledgements-This work was financially supported by the Dutch Technology Foundation (RGN 44.3462). The overall technical assistance of F.C. van Egmond, J. Honkoop and F. Mastik and the assistance of G. van Dijk of the Laboratory for Seismics and Acoustics of the Delft University of Technology for the construction of the modified intravascular transducer is acknowledged.

\section{REFERENCES}

Baptista J, Di Mario C, Ozaki Y, et al. Impact of plaque morphology and composition on the mechanisms of lumen enlargement using intracoronary ultrasound and quantitative angiography after balloon angioplasty. Am J Cardiol 1996;77:115-121.

Barzilai B, Saffitz JE, Miller JG, Sobel BE. Quantitative ultrasonic characterization of the nature of atherosclerotic plaques in human aorta. Circ Res 1987;60:459-463.

Bergel DH. The static elastic properties of the arterial wall. J Physio 1961;156:445-457.

Bridal SL, Fornès P, Bruneval P, Berger G. Parametric (integrated backscatter and attenuation) images constructed using backscattered radio frequency signals $(25-56 \mathrm{MHz})$ from human aortae in vitro. Ultrasound Med Biol 1997a;23:215-229.

Bridal SL, Fornès P, Bruneval P, Berger G. Correlation of ultrasonic attenuation (30 to $50 \mathrm{MHz}$ ) and constituents of atherosclerotic plaque. Ultrasound Med Biol 1997b;23:691-703.

Céspedes EI, Ophir J, Ponnekanti H, Maklad N. Elastography: Elasticity imaging using ultrasound with application to muscle and breast in vivo. Ultrason Imaging 1993;15:73-88.

Céspedes EI, Huang Y, Ophir J, Spratt S. Methods for estimation of subsample time delays of digitized echo signals. Ultrason Imaging 1995a;17:142-171.

Céspedes EI, Insana M, Ophir J. Lower bounds on strain estimation in elastography. IEEE Trans Ultrason Ferroelec Freq Contr 1995b;42: 969-972.

Céspedes EI, de Korte CL, van der Steen AFW, Norder B, te Nijenhuis $\mathrm{K}$. Tissue mimicking material and artifacts in intravascular elastic- ity imaging. IEEE Ultrasonics Symp Proceedings, San Antonio, TX, USA 1996; 1181-1184.

Céspedes EI, de Korte CL, van der Steen AFW. Echo decorrelation from displacement gradients in elasticity and velocity estimation. IEEE Trans Ultrason Ferroelec Freq Contr 1997; (Submitted).

Cheng GC, Loree HW, Kamm RD, Fishbein MC, Lee RT. Distribution of circumferential stress in ruptured and stable atherosclerotic lesions. A structural analysis with histopathological correlation. Circulation 1993;87:1179-1187.

de Korte CL, Céspedes EI, van der Steen AFW, Lancee CT. Image artefacts in intravascular elastography. IEEE EMBS Conference Proceedings, Amsterdam, The Netherlands, 1996; CD-rom paper no. 487

de Korte CL, Céspedes EI, van der Steen AFW, Lancee CT. Intravascular elasticity imaging using ultrasound: Feasibility studies in phantoms. Ultrasound Med Biol 1997;23:735-746.

Fitzgerald PJ, Yock, PG. Mechanisms and outcomes of angioplasty and atherectomy assessed by intravascular ultrasound imaging. J Clin Ultrasound 1993;21:579-588.

Gow BS, Taylor MG. Measurement of viscoelastic properties of arteries in the living dog. Circ Res 1968;23:111-122.

Gussenhoven EJ, van der Lugt A, Pasterkamp G, et al. Intravascular ultrasound predictors of outcome after peripheral balloon angioplasty. Eur J Vasc Endovasc Surg 1995;10:279-288.

Hoeks APG, Brands PJ, Reneman RS. Assessment of the arterial distension waveform using Doppler signal processing. J Hypertens 1992;10(S6):s19-s22.

Honye J, Mahon DJ, Jain A, et al. Morphological effects of coronary balloon angioplasty in vivo assessed by intravascular ultrasound imaging. Circulation 1992;85:1012-1025.

IEEE Programs for digital signal processing. Algorithm 8.1. New York: IEEE Press, John Wiley \& Sons, 1979.

Isner JM, Rosenfield K, Losordo DW, et al. Combination of balloonultrasound imaging catheter for percutaneous transluminal angioplasty. Circulation 1991;84:739-754.

Landini L, Sarnelli R, Picano E, Salvadori M. Evaluation of frequency dependence of backscatter coefficient in normal and atherosclerotic aortic walls. Ultrasound Med Biol 1986;12:397-401.

Lee RT, Richardson SG, Loree HM, et al. Prediction of mechanical properties of human atherosclerotic tissue by high-frequency intravascular ultrasound imaging: An in vitro study. Arterioscler Thromb 1992;12:1-5.

Lee RT, Grodzinsky AJ, Frank EH, Kamm RD, Schoen FJ. Structure dependent dynamic mechanical behavior of fibrous caps from human atherosclerotic plaque. Circulation 1991;83:1764-1770.

Lee RT, Loree HM, Cheng GC, et al. Computational structural analysis based on intravascular ultrasound imaging before in vitro angioplasty: Prediction of plaque fracture locations. J Am Coll Cardiol 1993;21:777-782.

Mintz GS, Pichard AD, Kovach JA, et al. Impact of preintervention intravascular ultrasound imaging on transcatheter treatment strategies in coronary artery disease. Am J Cardiol 1994;73:423-430.

Mintz GS, Popma JJ, Pichard AD, et al. Arterial remodeling after coronary angioplasty. A serial intravascular ultrasound study. Circulation 1996;94:35-43.

Mozersky DJ, Sumner DS, Hokanson DE, Strandness DE. Transcutaneous measurement of the elastic properties of the humen femoral artery. Circulation 1972;46:948-955.

O'Donnell M, Eberle MJ, Stephens DN, et al. Synthetic phased arrays for intraluminal imaging of coronary arteries. IEEE Trans Ultrason Ferroelec Freq Contr 1997;44:714-721

Ophir J, Céspedes EI, Ponnekanti H, Yazdi Y, Li X. Elastography: A method for imaging the elasticity in biological tissues. Ultrason Imaging 1991;13:111-134.

Pagani M, Mirsky I, Baig H, et al. Effects of age on aortic pressure diameter and elastic stiffness-stress relationships in unanesthetized sheep. Circ Res 1979;44:420-429.

Patel DJ, Tucker WK, Janicki JS. Dynamic elastic properties of the aorta in radial direction. J Appl Physiol 1970a;28:578-582.

Patel DJ, Janicki JS. Static elastic properties of the left coronary 
circumflex artery and the common carotid artery in dogs. Circ Res 1970b;27:149-158.

Reneman RS, Merode T, Hick P, Muytjens AMM, Hoeks APG. Agerelated changes in carotid artery wall properties in men. Ultrasound Med Biol 1986;12:464-471.

Ryan LK, Foster FS. Tissue equivalent vessel phantoms for intravascular ultrasound. Ultrasound Med Biol 1997a;23:261-273.

Ryan LK, Foster FS. Ultrasonic measurement of differential displacement and strain in a vascular model. Ultrason Imaging 1997b;19:19-38.

Shapo BM, Crowe JR, Skovoroda AR, et al. Displacement and strain imaging of coronary arteries with intraluminal ultrasound. IEEE Trans Ultrason Ferroelec Freq Contr 1996;43:234-246.

Soualmi L, Bertrand M, Mongrain R, Tardif JC. Forward and inverse problems in endovascular elastography. Proceedings 23rd International Symposium Acoustic Imaging 1997; (In press).
Spencer T, Ramo MP, Salter DM, et al. Characterisation of atherosclerotic plaque by spectral analysis of intravascular ultrasound: An in vitro study. Ultrasound Med Biol 1997;23:191-203.

ten Hoff H, Korbijn A, Smit TH, Klinkhamer JFF, Bom N. Imaging artefacts in mechanically driven ultrasound catheters. Int $\mathbf{J}$ Card Imaging 1989;4:195-199.

The SHK, Gussenhoven EJ, Pieterman G, et al. Assessment of regional vascular distensibility in deceased iliofemoral arteries by intravascular ultrasound. Ultrasound Med Biol 1995;21:17-24.

Wilson LS, Neale ML, Talhami HE, Appleberg M. Preliminary results from attenuation-slope mapping of plaque using intravascular ultrasound. Ultrasound Med Biol 1994;20:529-542.

Wolverson MK, Bashiti HM, Peterson GJ. Ultrasonic tissue characterization of atheromatous plaques using a high resolution real time scanner. Ultrasound Med Biol 1983;9:599-609. 